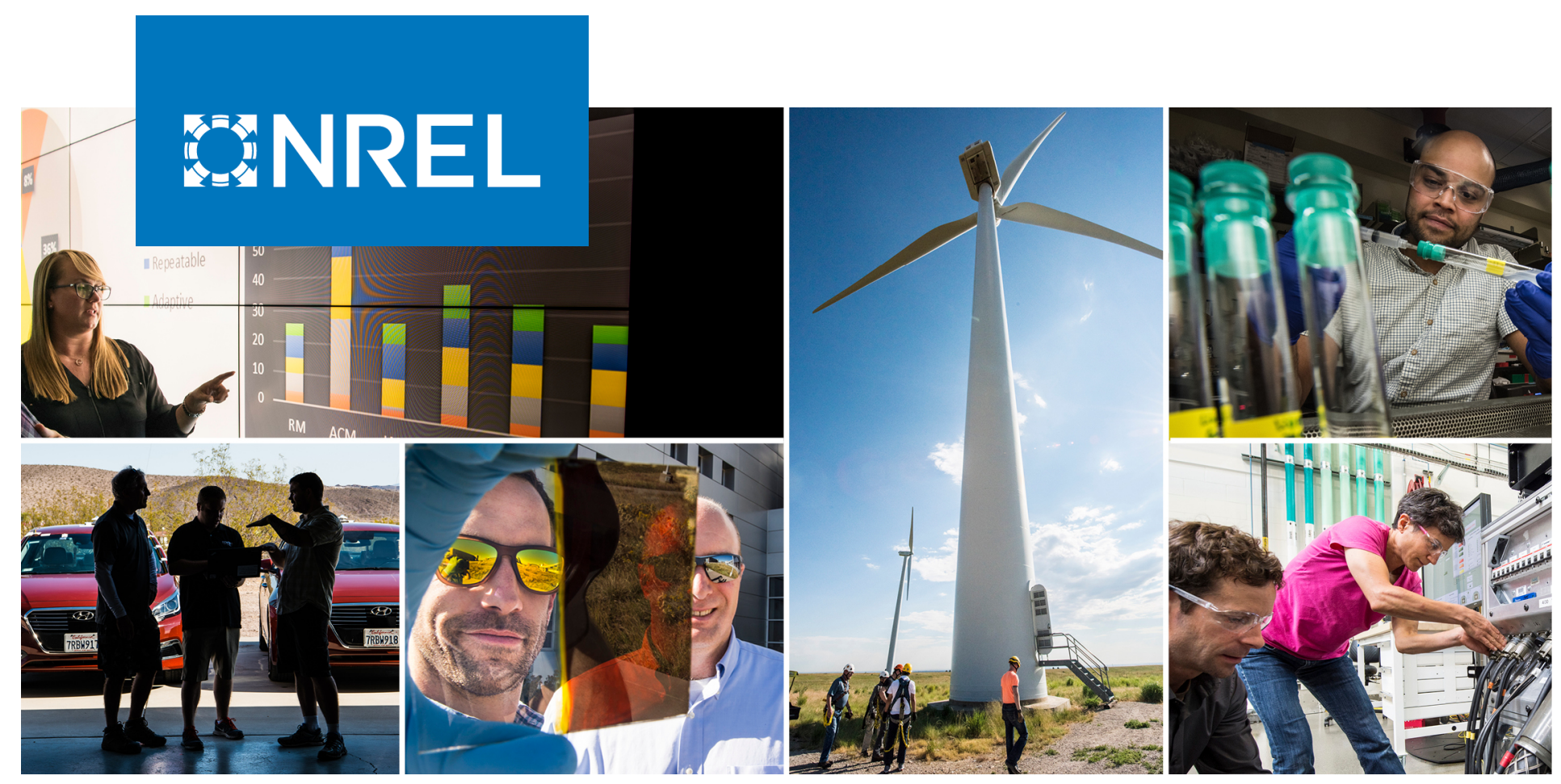

\title{
Using an Advanced Distribution Management System Test Bed to Evaluate the Impact of Model Quality on Volt/VAR Optimization
}

\section{Preprint}

Annabelle Pratt, ${ }^{1}$ Ismael Mendoza, ${ }^{1}$ Muhammad Usama Usman, ${ }^{1}$ Soumya Tiwari, ${ }^{1}$ Harsha Padullaparti, ${ }^{1}$ Murali Baggu, ${ }^{1}$ and Eric Lightner ${ }^{2}$

${ }^{1}$ National Renewable Energy Laboratory

2 U.S. Department of Energy

Presented at the 2020 IEEE Power and Energy Society Transmission and Distribution Conference and Exposition (IEEE PES T\&D)

October 12-15, 2020

NREL is a national laboratory of the U.S. Department of Energy Office of Energy Efficiency \& Renewable Energy

Operated by the Alliance for Sustainable Energy, LLC

This report is available at no cost from the National Renewable Energy Laboratory (NREL) at www.nrel.gov/publications.
Conference Paper

NREL/CP-5D00-74723

October 2020 


\title{
GNREL
}

\section{Using an Advanced Distribution Management System Test Bed to Evaluate the Impact of Model Quality on Volt/VAR Optimization}

\section{Preprint}

\author{
Annabelle Pratt, ${ }^{1}$ Ismael Mendoza, ${ }^{1}$ Muhammad Usama \\ Usman, ${ }^{1}$ Soumya Tiwari, ${ }^{1}$ Harsha Padullaparti, ${ }^{1}$ \\ Murali Baggu, ${ }^{1}$ and Eric Lightner ${ }^{2}$ \\ ${ }^{1}$ National Renewable Energy Laboratory \\ 2 U.S. Department of Energy
}

\section{Suggested Citation}

Pratt, Annabelle, Ismael Mendoza, Muhammad Usama Usman, Soumya Tiwari, Harsha Padullaparti, Murali Baggu, and Eric Lightner. 2020. Using an Advanced Distribution Management System Test Bed to Evaluate the Impact of Model Quality on Volt/VAR Optimization: Preprint. Golden, CO: National Renewable Energy Laboratory. NREL/CP-5D00-74723. https://www.nrel.gov/docs/fy21osti/74723.pdf.

(C) 2020 IEEE. Personal use of this material is permitted. Permission from IEEE must be obtained for all other uses, in any current or future media, including reprinting/republishing this material for advertising or promotional purposes, creating new collective works, for resale or redistribution to servers or lists, or reuse of any copyrighted component of this work in other works.

NREL is a national laboratory of the U.S. Department of Energy Office of Energy Efficiency \& Renewable Energy Operated by the Alliance for Sustainable Energy, LLC

This report is available at no cost from the National Renewable Energy Laboratory (NREL) at www.nrel.gov/publications.

Contract No. DE-AC36-08GO28308
Conference Paper

NREL/CP-5D00-74723

October 2020

National Renewable Energy Laboratory 15013 Denver West Parkway Golden, CO 80401

303-275-3000 • www.nrel.gov 


\section{NOTICE}

This work was authored by the National Renewable Energy Laboratory, operated by Alliance for Sustainable Energy, LLC, for the U.S. Department of Energy (DOE) under Contract No. DE-AC36-08GO28308. Funding provided by U.S. Department of Energy Office of Electricity Advanced Grid Research and Development Program, as part of the Grid Modernization Laboratory Consortium. The views expressed herein do not necessarily represent the views of the DOE or the U.S. Government.

This report is available at no cost from the National Renewable Energy Laboratory (NREL) at www.nrel.gov/publications.

U.S. Department of Energy (DOE) reports produced after 1991 and a growing number of pre-1991 documents are available free via www.OSTI.gov.

Cover Photos by Dennis Schroeder: (clockwise, left to right) NREL 51934, NREL 45897, NREL 42160, NREL 45891, NREL 48097, NREL 46526.

NREL prints on paper that contains recycled content. 


\section{Using an Advanced Distribution Management System Test Bed to Evaluate the Impact of Model Quality on Volt/VAR Optimization}

\author{
Annabelle Pratt, Ismael Mendoza, Muhammad Usama Usman, \\ Soumya Tiwari, Harsha Padullaparti, Murali Baggu \\ National Renewable Energy Laboratory \\ Golden, Colorado, USA \\ annabelle.pratt@nrel.gov
}

\author{
Eric Lightner \\ Advanced Grid Research \& Development \\ Office of Electricity, U.S. Department of Energy \\ Washington, D.C., USA
}

\begin{abstract}
In this paper, we present a test bed for evaluating existing and future advanced distribution management system (ADMS) applications in a realistic laboratory setting, including other utility management systems and field equipment. We present an example of using it to evaluate the impact of the ADMS network model quality on a Volt/ VAR optimization (VVO) application. The test bed integrates a commercial ADMS with a real-time simulation model of a utility distribution feeder. Representative power and controller hardware are integrated through hardware-in-the-loop (HIL) techniques. Initial results indicate that a higher model quality achieves the highest possible energy savings while avoiding voltage exceedances, whereas a lower model quality results in increased energy savings but at the expense of more voltage exceedances.
\end{abstract}

Keywords-advanced distribution management system (ADMS), power distribution system, hardware-in-the-loop (HIL) simulation, test bed, Volt/VAR optimization (VVO)

\section{INTRODUCTION}

Distribution system operators (DSOs) need to prepare for a future electric grid with increased penetrations of renewable and distributed energy resources (DERs), prosumers and microgrids, as well as new business models and services, some of which could be provided by third-party aggregators. This complex operating environment will require enhanced operating systems, platforms, and applications for the management of electricity. Advanced distribution management systems (ADMS) have emerged and offer a single software platform to provide traditional functions, such as supervisory control and data acquisition (SCADA), an outage management system (OMS), customer information system (CIS) and geographic information system (GIS). ADMS platforms also offer advanced functions, such as Volt/volt-ampere reactive (VAR) optimization (VVO); fault location, isolation, and system restoration (FLISR); and dynamic voltage regulation [1].

This work was authored by the National Renewable Energy Laboratory, operated by Alliance for Sustainable Energy, LLC, for the U.S. Department of Energy (DOE) under Contract No. DE-AC36-08GO28308. Funding provided by U.S. Department of Energy Office of Electricity Advanced Grid Research and Development program, as part of the Grid Modernization Laboratory Consortium. The views expressed in the article do not necessarily represent the views of the DOE or the U.S. Government. The U.S. Government retains and the publisher, by accepting the article for publication, acknowledges that the U.S. Government retains a nonexclusive, paid-up, irrevocable, worldwide license to publish or reproduce the published form of this work, or allow others to do so, for U.S. Government purposes.
Despite the promise of ADMS platforms to improve resilience and security and increase power quality and reliability [1], ADMS deployment is still in the early stages. One reason is that ADMS deployment requires a significant investment of time and funds by utilities, which in turn requires that the benefits the ADMS provides are well understood and quantified for the utilities. To address this challenge, the U.S. Department of Energy (DOE) Office of Electricity has invested in the development of a vendor-neutral ADMS test bed as part of the Grid Modernization Initiative [2]. The test bed provides utilities and vendors the opportunity to understand the benefits of specific applications for a specific distribution system under a wide range of conditions that can be simulated and emulated in a laboratory environment. Another advantage of a laboratory test bed is that it can be used to evaluate the effectiveness of ADMS applications on a futuristic model of a utility feeder. For example, a feeder that currently has a low penetration of photovoltaic (PV) generation can be modeled with the level of PV penetration that is forecasted in 5, 10, or 20 years. Similarly, more efficient and flexible heating and cooling systems or increased penetrations of electric vehicles (EVs) can be modeled as well as emerging controls and management systems.

We developed a collaborative approach involving both the utility and ADMS vendor to define the specific conditions to simulate and analysis to perform. We refer to the combination of these as use cases; further details on potential use cases are presented in [3]. This paper presents a use case developed in collaboration with Xcel Energy, an investor-owned utility, and Schneider Electric, the ADMS vendor selected by Xcel Energy. This use case studies the impact of the quality of the network model within an ADMS on its VVO application.

Section II of this paper provides an overview of the ADMS test bed and the model quality use case. Sections III and IV present how the test bed is configured for this use case and experimental results obtained from the ADMS test bed, respectively. Section $\mathrm{V}$ concludes with a discussion on potential future use cases that can support the power industry in managing power systems with high penetrations of DERs.

\section{ADMS TEST BED OVERVIEW}

The ADMS test bed used in the paper is hosted by the National Renewable Energy Laboratory's (NREL's) Energy Systems Integration Facility (ESIF). The test bed consists of software and hardware elements to realistically represent a power distribution system. 
Advanced Distribution Management System (ADMS)

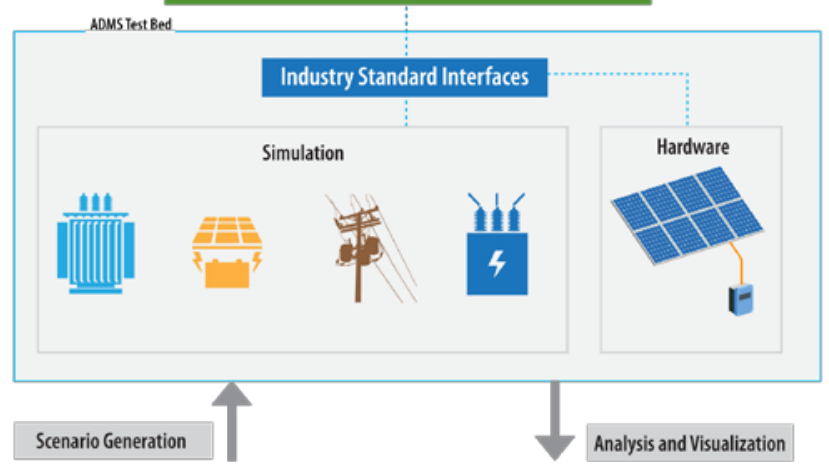

Fig. 1. Overview of the ADMS test bed.

The test bed uses industry standard communications protocols to interface the ADMS with software and hardware components. Fig. 1 shows an overview of the test bed. The main elements are a multi-timescale simulation, controller and power hardware that can be interfaced with the software simulation through the use of hardware-in-the-loop (HIL) techniques, and industry-standard communications interfaces.

The multi-timescale simulation of the power distribution system is the central element of the test bed. The network model will consist mostly of feeders selected by the utility; however, we can also use synthetic feeders, i.e., anonymized, realistic feeders, such as those from the Advanced Research Projects Agency-Energy GRID DATA program or representative feeders provided by the Electric Power Research Institute. This might be particularly useful for a vendor-driven use case with the intent of showing the value of a new application.

The multi-timescale simulation currently includes both an open-source, quasi-steady-state time-series power system simulator, OpenDSS, with a typical time step of 1 minute; and a faster power system simulator executed on an OPAL-RT digital real-time simulator. On the OPAL-RT, either a transient stability simulator (using a phasor-domain solver), ePHASORSIM, with a typical time step of 10 milliseconds, or an electromagnetic transient (EMT) simulator, eMEGASIM, with a typical time step of 100 microseconds, can be used to simulate the power system. We can simulate the entire network in OpenDSS or in Opal-RT, or we can cosimulate the network with part of it modeled in OpenDSS and part of it modeled in Opal-RT. For cosimulation, we developed software to coordinate the ePHASORSIM, eMEGASIM, and OpenDSS simulators, and it uses the Hierarchical Engine for Large-scale Infrastructure CoSimulation (HELICS), an open-source cyber-physical-energy cosimulation framework for electric power systems developed through the Grid Modernization Initiative of DOE [4]. We anticipate that we would integrate different power system simulation tools, including other digital real-time simulators, with the test bed to support future use cases.

The other essential element of the test bed is the ability for all the simulated devices that need to be interfaced with the ADMS to communicate using an industry-standard communications protocol. Simulated devices in ePHASORSIM or eMEGASIM can communicate through libraries available on the OPAL-RT platform. The OpenDSS simulator does not support communications protocols, and therefore we developed software that can exchange data between the ADMS and OpenDSS using industry-standard communications protocols.

It is also possible to integrate controller and/or power hardware with the simulation using HIL techniques. Controller hardware that might be integrated with the test bed include controllers for capacitor banks, voltage regulators, and load tap changers (LTCs); protection relays; and genset controllers. Power hardware might include PV or battery inverters, EVs, or conventional generators. Finally, other utility management systems - such as a transmission energy management system, DER management system (DERMS), microgrid controller, or building automation system - can also be incorporated into the experiment as needed for a specific use case.

For each use case, a set of scenarios is generated that includes loading and solar insolation conditions, DER penetration levels, and/or faults. These scenarios are then simulated and can be observed using real-time visualization tools developed for the test bed, and the data are stored to enable analysis of them.

\section{Model QuAlity UsE CASE DESCRIPTION}

We present a use case here that was developed in partnership with our utility partner, Xcel Energy, which is in the process of deploying an ADMS provided by Schneider Electric [5]. Xcel Energy plans to use the ADMS VVO application to reduce the energy use and potentially monthly bills for their customers. The VVO application, when configured for energy conservation, aims to reduce energy use by flattening the voltage profile and reducing voltages across the feeder as much as possible while avoiding voltage exceedances. This is sometimes referred to as conservation voltage reduction (CVR).

This can be achieved with VVO configured to utilize only telemetry data or to utilize both the network model and telemetry data. With the penetration of renewable generation continuing to increase in distribution systems, we can no longer assume that the voltage is lower the further a point is located from the feeder head. This can limit the effectiveness of relying only on voltage measurements at a number (typically 10 to 20) of pre-selected points at the edge of a feeder. Using a network model within the VVO application can provide additional insight into the voltage profile, especially for feeders with a high penetration of renewable generation. Therefore, the VVO application was configured for this research to utilize a network model and realtime measurements to optimize the set points of legacy voltage control devices, such as voltage regulators and capacitor banks. In the future, with increased penetrations of smart inverters for DERs, VVO applications might also optimize DER power set points or interface with DERMS that provide voltage support services.

If a utility/DSO does not have a high level of confidence in the accuracy of the network model used by the ADMS, they might use more conservative constraints, keeping the voltages in the feeder higher to avoid undervoltage exceedances. This approach would result in reduced energy savings. Alternatively, a utility might invest in data remediation (specifically data cleansing) to gain more confidence in the model and operate with less conservative constraints and potentially increase 
energy savings. Data remediation is an expensive and timeconsuming process, and results of our work will help Xcel Energy understand how much data remediation is required to achieve the desired result from their ADMS investment.

Preliminary results for the impact of model quality were obtained by simulating six Xcel Energy feeders-four urban and two rural-using the ADMS training simulator as a proxy for the real feeders. To evaluate the impact of model quality, four different network models were developed for each feeder for use within the ADMS. The lowest model quality level (Q1) contains base-level data extracted from the Xcel Energy GIS adjusted to provide power flow convergence. The next level of model quality (Q2) adds field verification at selected locations of capacitor, voltage regulator, and recloser attributes. The next quality level (Q3) adds phasing information at selected locations. The highest quality level (Q4) adds distribution transformer attributes, identifying new assets not shown in the GIS and identifying assets that no longer exist in the field.

In addition, the number and location of telemetry points were adjusted in the ADMS training simulator to four different measurement density levels. The lowest level of measurement density (D1) provides only feeder-head measurements. The next level (D2) adds measurements at capacitor banks and reclosers. The next level (D3) adds 10 advanced metering infrastructure (AMI) sensors, and the highest level (D4) adds 10 more AMI sensors. Four different levels of model quality and four different levels of measurement density result in 16 possible combinations that were simulated with three different loading conditions within the ADMS training simulator. The results of these simulations will be presented in another paper.

In summary, results show that the feeder characteristics greatly influence the impact of different model quality levels. Rural feeders show a greater impact on energy consumption for different levels of model quality compared to urban feeders. This is because the urban feeders are relatively new and have been field-verified in recent years, whereas the rural feeders are older and their data have not been updated as recently.

The SCADA points in the ADMS training simulator were set up with a dead band that would report a measurement value only when the current measurement exceeded the dead band value. This provided a limited number of data points (typically less than 50 per day) for our analysis. In addition, data are available for analysis only from the telemetry points defined by the different levels of measurement density, which is very limited for the lower levels of measurement density.

As discussed in the next section, simulation of a feeder using the ADMS test bed provides much more granular results, with a simulation time step of 1 minute. Further, we can monitor voltages at several end-of-line locations and power injections by all PV systems on the feeder when using the ADMS test bed. We can also integrate actual hardware and evaluate the interoperability of different control systems and devices with the ADMS test bed.

\section{ADMS Test Bed Setup For Model Quality Use CaSE}

Fig. 2 shows the ADMS test bed setup for the model quality use case. We simulate one urban Xcel Energy feeder using the multi-timescale simulation capability.

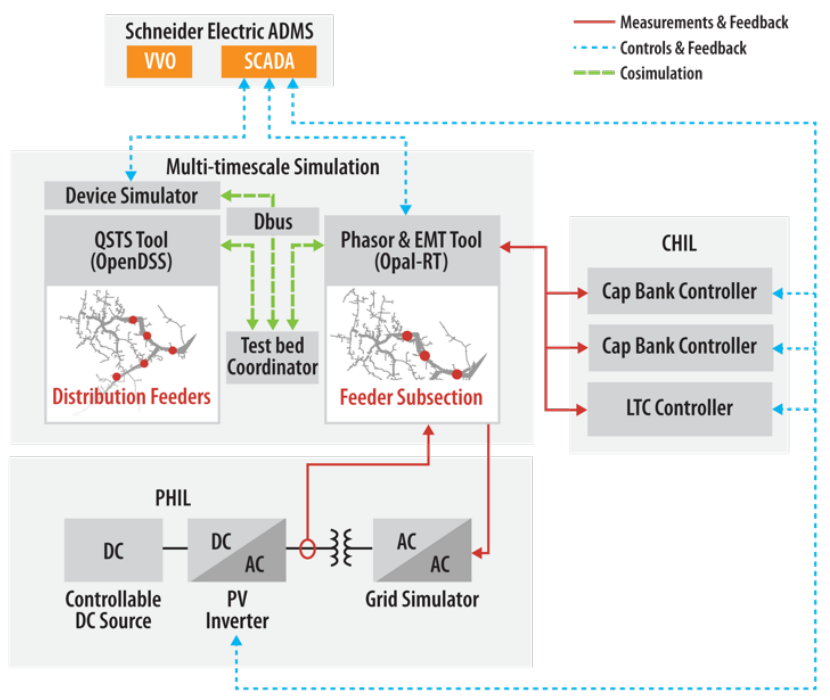

Fig. 2. Diagram of the ADMS test bed setup for the model quality use case

The feeder has a peak load of about $12 \mathrm{MW}$, an LTC at the feeder head, and four capacitor banks. Part of the feeder is simulated in OpenDSS, and the rest of the feeder is simulated in ePHASORSIM. OPAL-RT's eMEGASIM is used to interface to the hardware, which consists of a 12-kVA three-phase PV inverter, two capacitor bank controllers, and an LTC controller.

The capacitor bank and LTC controllers are interfaced with the power system simulations through the digital-to-analog and analog-to-digital interfaces provided on the OPAL-RT platform [6]. The controllers receive low-voltage signals representing simulated voltages and currents from OPAL-RT and issue control set points that are extracted and fed back to the simulated equipment. The PV inverter power hardware is interfaced with the power system simulations through a grid simulator (a controllable AC power source). The grid simulator receives a low-voltage signal from the OPAL-RT representing the simulated voltage at the node in the power system simulation where the power hardware is connected, and it amplifies it to (nominally) the rated voltage of the power hardware [7]. The DC power input to the PV inverter is supplied by a PV simulator, a specialized controllable DC source that can emulate a PV panel. Fig. 3 shows a photograph of the ADMS test bed setup for the model quality use case in NREL's ESIF [8].

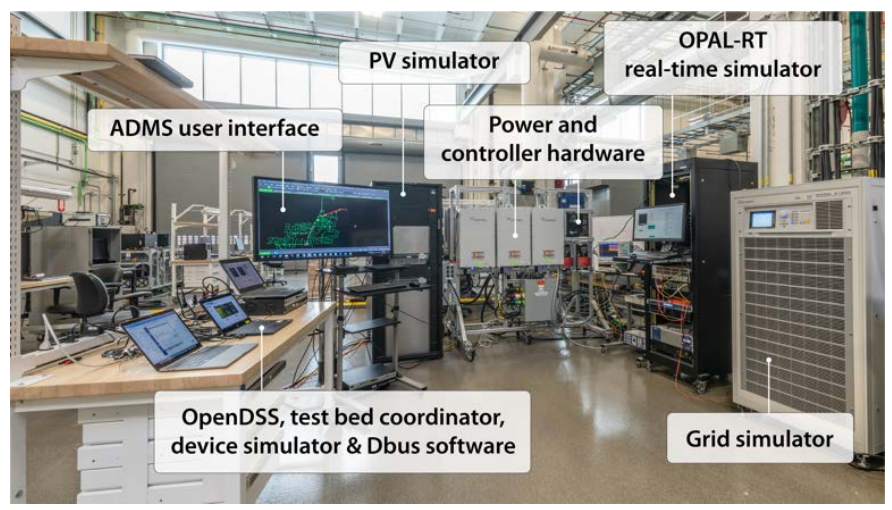

Fig. 3. Photograph of the ADMS test bed setup for the model quality use case. Photo by Joshua Bauer, NREL 
The ADMS uses the DNP3 protocol to communicate with all the simulated and actual devices in the test bed. OPAL-RT supports the DNP3 protocol, but OpenDSS does not. Therefore, device simulator software was developed to provide a DNP3 interface with OpenDSS. The device simulator software uses a data bus (Dbus) to exchange data with the test bed coordinator, which interfaces with OpenDSS. The device simulator software also includes the control functionality of a capacitor bank controller, and this is used for the two simulated capacitor bank controllers.

\section{EXPERIMENTAL RESUlts}

We present results here from simulations that we performed on the ADMS test bed with two different network model qualities. We will perform additional experiments with other network model qualities and different measurement densities in the future. The VVO primary objective is set up for energy conservation, which is one of the planned objectives for use in the field. The VVO application aims to achieve this objective by decreasing the voltages across the feeder while avoiding voltage exceedances. We consider a voltage below 0.95 p.u. or above 1.05 p.u. to be an exceedance, based on the ANSI C84.1 industry standard. The VVO closed-loop application was configured to run every 15 minutes to emulate field settings, performing state estimation and calculating the optimal LTC voltage set point and the optimal states (open or closed) of the capacitor banks to achieve the desired objective. (It can also be set up to run at shorter time intervals and when voltage violations occur to make it event-triggered.) We use a load profile provided by Xcel Energy that corresponds to a winter day, which is a low-load scenario for the feeder, and a solar insolation profile for an average clear winter day that peaks at about 12:30.

The simulated scenarios consisted of two different levels of model quality within the ADMS, in the following two combinations: Q1/D1 and Q4/D1. These were chosen based on the results from the simulations using the ADMS training simulator, as the two use cases in which the impact of the model quality can be best assessed.

A baseline simulation was also performed that excluded the ADMS and for which the capacitor bank controllers and LTC controller were set up in local control mode with the same settings as those used in the field. The LTC controller voltage set point was $124.3 \mathrm{~V}$ (1.035 p.u.), and the bandwidth was 3.5 V. The capacitor bank controllers had a close threshold of 0.99 p.u. and an open threshold of 1.04 p.u. The results obtained from the baseline simulation served as the base to compare the energy use when the ADMS is incorporated into the test.

All the simulations were started with all the capacitors in a closed position and with the baseline LTC voltage set point. The priority of the LTC is set higher than the capacitor banks in the configuration of the ADMS, i.e., the VVO application lowers the voltage by changing the tap position of the LTC and utilizes the capacitors to flatten the voltage profile for additional voltage reduction. When a voltage exceedance occurs, the LTC is the first choice to resolve it, and if this is not possible, the VVO changes the capacitor bank states. The experiments were run from 15:00 to 13:30 the next day to provide data for almost 24 hours while allowing time to set up the simulations on consecutive days. Data were logged once per minute from
OpenDSS and twice per second from ePHASORsim, starting at 15:30, and then all data were normalized to 1-minute intervals.

Fig. 4 shows the active power demand at the substation. It peaks at 7.3 MW for the baseline and is reduced to $6.82 \mathrm{MW}$ and 6.93 MW for Q1/D1 and Q4/D1 respectively by the VVO application. The power reduction is achieved by reducing voltages across the feeder. Fig. 5 shows a representative end-ofline voltage. The voltage is higher in the baseline scenario because the ADMS is not incorporated and the LTC is in local control mode with a voltage set point of $1.035 \mathrm{p}$.u. In the other scenarios, where the ADMS is incorporated, the LTC voltage set point is reduced to achieve the configured VVO objective of energy conservation.

This is shown in Fig. 6, which shows the LTC tap position corresponding to different scenarios. The initial tap position at the start of the experiment is 0 . The tap position stayed constant for the baseline because the voltage always remained within the defined bandwidth of $124.3 \mathrm{~V} \pm 1.75 \mathrm{~V}$ at the secondary side of the LTC. In all other scenarios, the VVO recalculated the LTC set point every 15 minutes. Fig. 7 and Fig. 8 show the maximum, minimum and mean of 75 end-of-line voltages collected from various parts of the feeder simulated in ePHASORsim and OpenDSS. Fig. 7 shows that the VVO reduced the voltages for Q4/D1 compared to the baseline while avoiding voltage exceedances. Fig. 8 show that the voltages were also lower for Q1/D1, but with voltage exceedances.

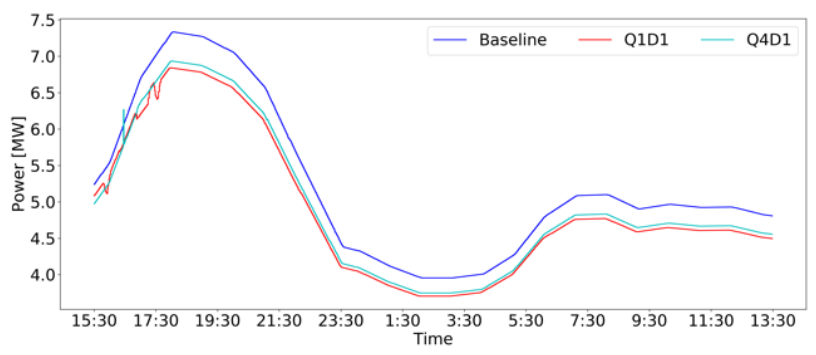

Fig. 4. Active power demand at the feeder head Figure

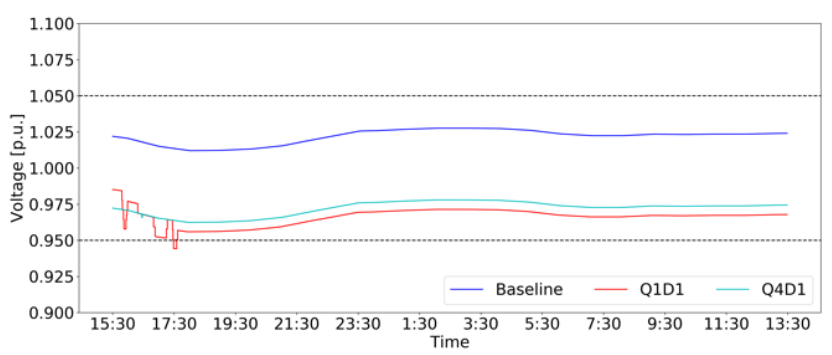

Fig. 5. A representative end-of-line voltage

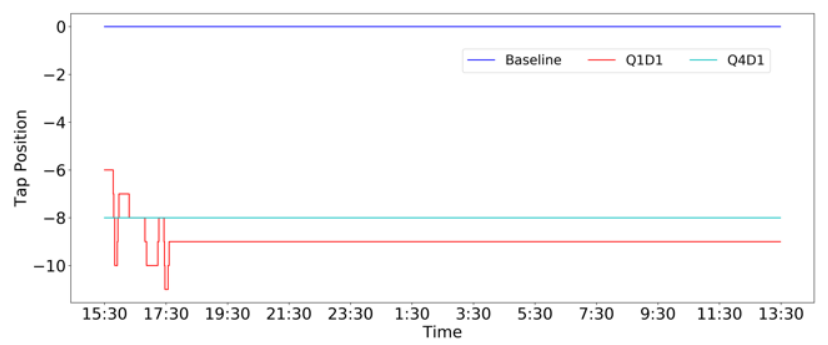

Fig. 6. LTC tap position 


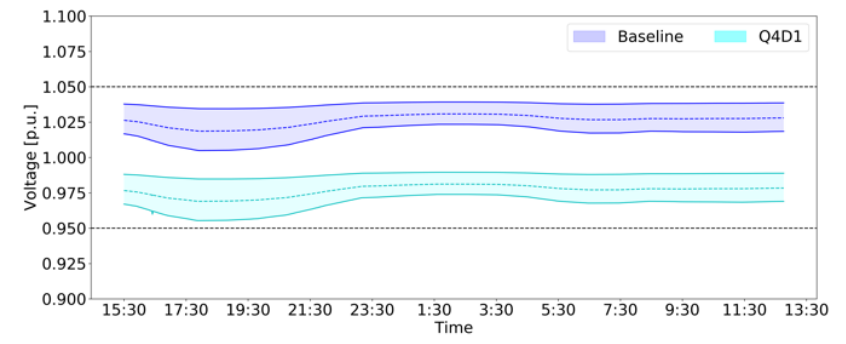

Fig. 7. Maximum, minimum and mean of end-of-line voltages for baseline and Q4/D1

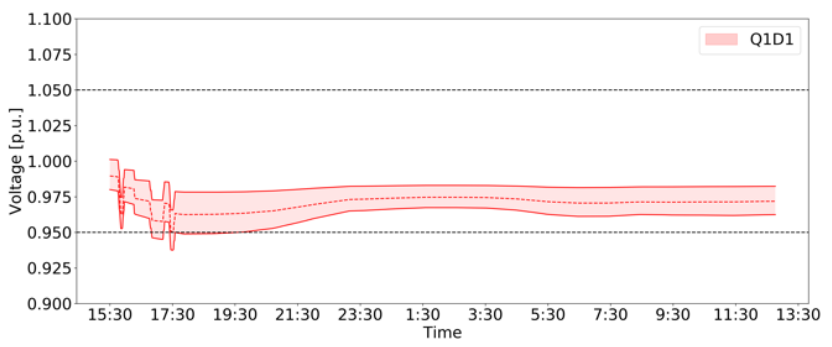

Fig. 8. Maximum, minimum and mean of end-of-line voltages for Q1/D1

Table I shows the total number of voltage exceedances along with energy savings compared to the baseline and the number of LTC tap position changes. There are no capacitor state changes because the capacitors remained in their initial state for all simulations. The results in Table I are calculated from data logged between 15:30 and 13:30 the next day (i.e., 22 hours).

The LTC tap position never changed for the baseline; and for Q4/D1, the tap changed from 0 to -8 in the first 30 minutes of the experiment, during which time no data were logged, and then stayed at -8 for rest of the experiment. Therefore, no tap changes were recorded for Q4/D1. For Q1/D1, there were 16 tap changes during the observed time. The maximum energy savings, compared to the baseline results, is observed for the Q1/D1 simulation. This is because the VVO reduces the voltages the most for Q1/D1, as shown in Fig. 6 and Fig. 8; however, Q1/D1 also exhibits the highest number of voltage exceedances, calculated by adding the number of voltage exceedances every minute for each observed end-of-line voltage. The energy savings were lowest for Q4/D1, but no voltage exceedances were observed.

TABLE I SUMMARY OF EXPERIMENTAL RESULTS

\begin{tabular}{|l|c|c|c|}
\hline & $\begin{array}{c}\text { Number of LTC } \\
\text { tap changes }\end{array}$ & $\begin{array}{c}\text { Energy savings } \\
\text { (\%) }\end{array}$ & $\begin{array}{c}\text { Number of voltage } \\
\text { exceedances }\end{array}$ \\
\hline Baseline & 0 & N/A & 0 \\
\hline Q1/D1 & 16 & 6.4 & 784 \\
\hline Q4/D1 & 0 & 5.3 & 0 \\
\hline
\end{tabular}

Initial results therefore indicate that a higher model quality (Q4) achieves the highest possible energy savings while avoiding voltage exceedances, whereas a lower model quality (Q1) results in increased energy savings but at the expense of more voltage exceedances. It should be noted that the network model simulated in the test bed are very similar to the highest ADMS network model quality (Q4), since both draw on the most remediated data provided by Xcel. Therefore, further study is warranted.

\section{CONCLUSIONS AND FUTURE WORK}

The rapidly evolving electric power system creates new challenges and opportunities as the deployment of DERs continues. ADMS are poised to serve a key role in ensuring the reliable operation of the future grid, but utilities need to be able to quantify the potential for performance improvement on their specific systems to deploy ADMS. We propose that evaluating existing and future ADMS applications in a realistic laboratory setting - including other utility management systems and field equipment-will allow utilities a cost-effective way to obtain the insight that they need and as a result will accelerate the deployment of ADMS. We present an ADMS test bed that has been developed at NREL with funding from DOE that can be used by utilities, vendors, and researchers.

In this paper, we presented initial results from the ADMS test bed to evaluate the impact of ADMS network model quality on the performance of a VVO application for an urban feeder. We will conduct more experiments for this use case, including experiments with different model qualities and measurement densities, as well as with heavy-load conditions. Next, we will use the ADMS test bed to evaluate the coordinated operation of DERMS and ADMS for peak load reduction. We anticipate using the test bed to quantify the operational benefits of a wide range of applications for specific utilities, for example, FLISR and VVO for feeders with very high penetrations of PV and/or EVs or that have microgrids connected.

\section{ACKNOWLEDGMENTS}

The authors thank Xcel Energy for providing data and Schneider Electric for supporting the ADMS setup at NREL and data analysis.

\section{REFERENCES}

[1] Voices of Experience: Insights into Advanced Distribution Management Systems, U.S. Department of Energy, February 2015.

[2] https://www.smartgrid.gov/files/ADMS_2-11.2015_revised.pdf

[3] S. Veda, H. Wu, M. Martin and M. Baggu, "Developing Use Cases for the Evaluation of ADMS Applications to Accelerate Technology Adoption," IEEE Green Technologies Conference (GREENTECH), March 2017.

[4] B. Palmintier, D. Krishnamurthy, P. Top, S. Smith, J. Daily and J. Fuller, "Design of the HELICS high-performance transmission-distributioncommunication-market co-simulation framework," Workshop on Modeling and Simulation of Cyber-Physical Energy Systems (MSCPES), April 2017.

[5] S. Veda, M. Baggu and A. Pratt, "Defining a Use Case for ADMS Testbed: Data Quality Requirements for ADMS Deployment," IEEE Conference on Innovative Smart Grid Technologies (ISGT), Feb 2019.

[6] J. Wang, B. Miller, A. Pratt, J. Fossum, T. Bialek and S. Mason, "Diesel Generator Controller Evaluation via Controller-Hardware-in-the-Loop for Various Microgrid Operation Modes," IEEE Conference on Innovative Smart Grid Technologies (ISGT), Feb 2019.

[7] J. Wang, A. Pratt, J. Fossum and M. Baggu, "Development of Application Function Blocks for Power-Hardware-in-the-Loop Interface Algorithm for Testing Grid-Connected Inverters," IEEE 9th International Symposium on Power Electronics for Distributed Generation Systems (PEDG), June 2018, Charlotte, NC, USA.

[8] A. Pratt, M. Baggu, F. Ding, et al, "A Test Bed to Evaluate Advanced Distribution Management Systems for Modern Power Systems," IEEE EUROCON 2019. 